\title{
Observation of the Dynamical Inversion of the Topological Charge of an Optical Vortex
}

\author{
Gabriel Molina-Terriza, Jaume Recolons, Juan P. Torres, and Lluis Torner \\ Laboratory of Photonics, Department of Signal Theory and Communications, Universitat Politecnica de Catalunya, \\ Gran Capitan UPC-D3, Barcelona, ES 08034, Spain \\ Ewan M. Wright \\ Optical Sciences Center, University of Arizona, Tucson, Arizona 85721
}

(Received 10 January 2001; published 22 June 2001)

\begin{abstract}
We report what is believed to be the first detailed experimental observation of the dynamic inversion of the topological charge of an optical vortex under free-space propagation. The vortex self-transformation occurs through continuous deformation of the noncanonical strength of the corresponding screw wave front dislocation, and is mediated by the occurrence of an extremely sharp turn in a Berry vortex trajectory, which observed at a Freund critical foliation appears as an edge-line dislocation orthogonal to the propagation direction, at a crucial point of the light evolution.
\end{abstract}

DOI: 10.1103/PhysRevLett.87.023902

PACS numbers: 42.25.-p, 42.65.-k

Topological deformation of fields is at the heart of the description of a vast variety of natural phenomena ranging from cosmology, to oceanography, chemistry, and biosciences. Its most extreme, yet ubiquitous manifestation occurs when the field is so strongly folded that it bends back upon itself to form a topological dislocation, or defect [1-5]. Screw dislocations, or vortices, are a common defect type. The multiplicity of the folding around the defect determines its topological charge. The net charge within a region of space must be conserved under continuous evolution provided that no charges enter or leave the region [6]. Therefore, dynamic vortex creation typically occurs through the nucleation of twin pairs of opposite charge. However, here we uncover a striking mechanism that inverts the topological charge of a single vortex under propagation. This vortex self-transformation is mediated by the occurrence of an extremely sharp turn in a Berry vortex trajectory $[7,8]$, which in the experimental plane of observation appears as an edge-line dislocation orthogonal to the light propagation direction, at a crucial point of the light evolution. Observations were performed with vortices nested in light beams [5-14]. The phenomenon can have direct implications to quantum photon states with angular momentum $[15,16]$, to superfluidity $[17,18]$, and to weakly interacting Bose-Einstein condensates (BEC's) $[19,20]$, and is believed to be generic to diverse vortex entities.

The key ingredient of the new phenomenon is the existence of vortices with intrinsic spatial structure [6], hereafter referred to as noncanonical vortices. In the case of optical beams, vortices are spiral phase ramps around a dark spot where the phase of the wave is undefined and thus the light intensity vanishes. The slowly varying field envelope $E$ of a single-charge vortex nested in a paraxial light beam propagating along the $Z$ axis has the form

$$
E=\left\{x-x_{1}(z)+i A(z)\left[y-y_{1}(z)\right]\right\} F(x, y, z),
$$

where $\left(x_{1}, y_{1}\right)$ stands for the vortex location in the $X-Y$ plane and $F$ is the host beam. The value of the complex quantity $A$ determines the noncanonical strength of the vortex: Only when $A= \pm 1$ does the vortex have a traditional, or canonical, structure. Vortices are located at the complex zeros of $E$. In the case of canonical vortices, the lines in the transverse plane where the real and imaginary parts of $E$ vanish cross at right angles. When $A \neq \pm 1$, the intensity pattern of the light beam $E$ has no radial symmetry around the vortex core, and at the location of the phase dislocation the real and imaginary parts of $E$ cross each other at general angles. The light intensity and phase structure of a single-charge noncanonical vortex with strength $A=1+i$ are shown in Fig. 1. The sign of the topological charge of the vortex, given by the circulation of the gradient of the phase of $E$ around the dislocation, is given by the sign of $\operatorname{Re}(A)$. Therefore, a family of different noncanonical vortices that carry the same topological charge can be constructed by varying the complex value of $A$ while keeping the sign of $\operatorname{Re}(A)$ fixed.

However, the dynamical evolution followed by the vortices, including the value of its topological charge, can depend critically on the noncanonical strength of the dislocations. In particular, self-inversion of the topological charge of a noncanonical vortex can occur. Furthermore, in contrast to traditional views, such phenomenon can take place in a variety of physical settings, including properly tailored light propagation in vacuum, counterintuitive though it might seem. The principle of the experimental realization of the dynamic inversion of the topological charge of a vortex is illustrated by the paraxial evolution of a single-charge vortex nested in the elliptical Gaussian beam $F=C(z) \exp \left[i x^{2} / q_{x}(z)+i y^{2} / q_{y}(z)\right]$, where $C(z)$ is the complex beam amplitude and $q_{x}(z)$ and $q_{y}(z)$ are the complex beam widths. In free space, the beam widths evolve as $q_{x}=2\left(z-z_{x}\right)-i w_{0 x}^{2}, q_{y}=$ $2\left(z-z_{y}\right)-i w_{0 y}^{2}$, where $z_{x}$ and $z_{y}$ stand for the locations 
(a)

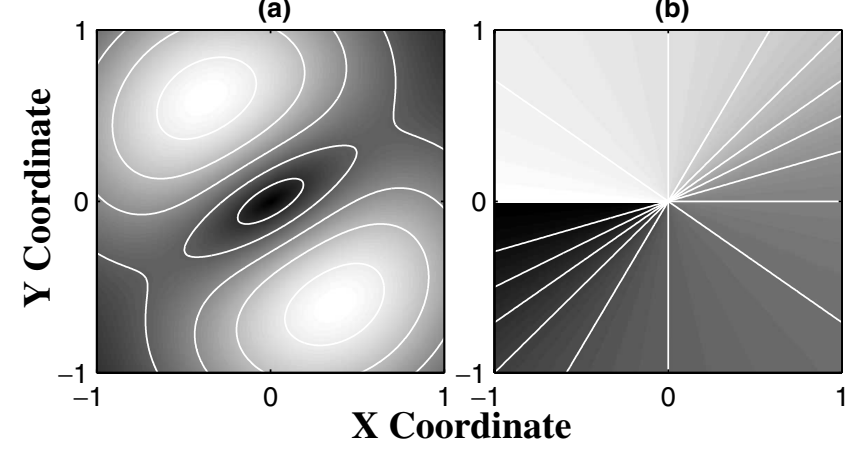

FIG. 1. Core of a noncanonical vortex with strength $A=$ $1+i$. (a) Amplitude. (b) Phase. Contour lines are spaced 0.11 dimensionless units in (a), and $\pi / 8$ units in (b).

of the beam waists along the propagation axis $Z$ and $w_{x}$, $w_{y}$ are the corresponding waists. Under such conditions, one finds that the noncanonical strength of the vortex varies during propagation according to the expression [21]

$$
A(z)=A_{0} \frac{2\left(z-z_{x}\right)-i w_{0 x}^{2}}{2\left(z-z_{y}\right)-i w_{0 y}^{2}},
$$

where $A_{0}$ is a constant. The vortex moves during propagation and it is located at the transverse coordinates that satisfy

$$
\frac{x_{1}(z)}{q_{x}}+i A_{0} \frac{w_{0 y}^{2}}{w_{0 x}^{2}} \frac{y_{1}(z)}{q_{y}}=\frac{x_{1,0}}{i w_{0 x}^{2}} .
$$

When $z_{x}=z_{y}$ and $w_{0 x}=w_{0 y}, A(z)$ is constant during propagation and hence so is the topological charge of the vortex. Nevertheless, as shown in Fig. 2a, this is not the case when the noncanonical character of the vortex is taken into account, and the charge of the vortex inverts sign. When the vortex is located exactly on axis (i.e., $x_{1,0}=0$ ), the inversion of the vortex charge occurs through a vortex trajectory [7] orthogonal to the light propagation direction (Fig. 2c). In the experimental plane of observation, which corresponds to Freund's critical foliation for this case [8], such trajectory appears as an edge-line dislocation. When either $x_{1,0} \neq 0$ or the beam is not exactly elliptical, as is the case in any experiment, the vortex trajectory is predicted to feature two extremely sharp turns towards and from the transverse infinity (Fig. 2d). An analogous behavior as above is obtained in more complex linear and nonlinear media, where, in general, the vortex evolution can be traced by solving numerically the paraxial wave equation, and accurate approximations of the evolution of $A(z)$ can be derived using multiple-scale expansion techniques around the vortex core $[21,22]$. As an illustrative example with relevance to evolution of vortices in trapped weakly interacting Bose-Einstein condensates [19,20], shown in Fig. $2 b$, is the evolution of an initially canonical vortex in an asymmetric graded-index (GRIN) linear medium (the GRIN medium plays the role of a magnetic trap for the BEC). In this case, the charge of the noncanonical vortex is predicted to change periodically along propagation.

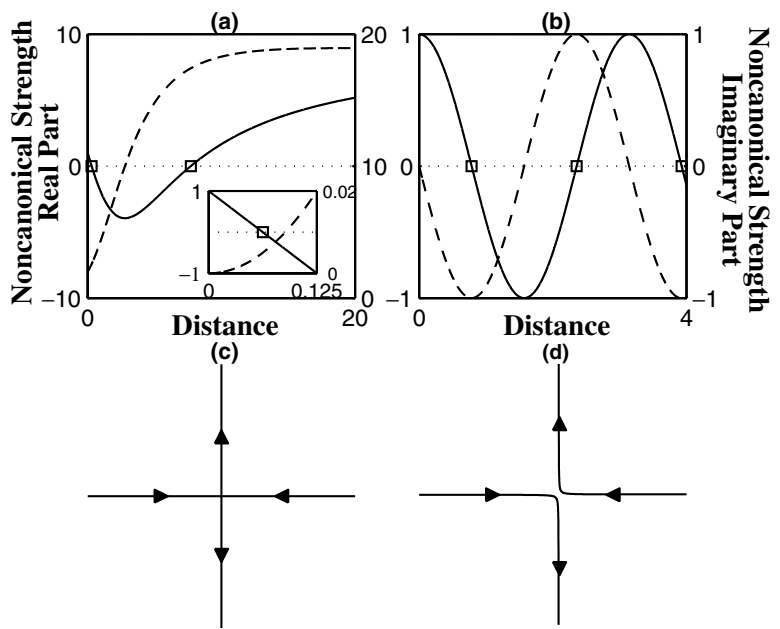

FIG. 2. Predicted evolution of the noncanonical strength of a vortex under different propagation conditions. Continuous and dashed lines show the evolution of the real and imaginary parts of $A$, respectively. Axes are in dimensionless units. The squares highlight the critical propagation distances where the inversion of the topological charge of the vortex occurs. (a) Evolution of a noncanonical vortex with $A(0)=1+2 i$, nested in an elliptical Gaussian beam with $w_{0 x}=1 w_{0 y}=3$. Inset: Predicted evolution of the noncanonical strength of the vortex under the conditions of our experimental setup. (b) Evolution of an input canonical vortex nested in an elliptical beam propagating in a GRIN medium with refractive index $n(x, y)=2 x^{2}+8 y^{2}$. The widths of the Gaussian beam are the stationary modes of the medium: $w_{x}=1, w_{y}=1 / \sqrt{2}$. (c),(d) Sketch of the Berry trajectories followed by on-axis and slightly off-axis vortices, respectively, when they undergo a topological charge inversion.

The experimental realization of an evolving noncanonical vortex can be performed in different settings. In an effort to highlight the universality of the phenomenon and to rule out complexity that might make the origin of the observations controversial, we performed our experiment to realize the evolution shown in Fig. 2a using an astigmatic optical scheme in free space. A linearly polarized, collimated light beam produced by a He:Ne laser at a wavelength $632.8 \mathrm{~nm}$ was split into two beams. Single-charge screw wave front dislocations were nested in one of the beams by using computer-generated holograms [11]. We used off-axis holograms, which were designed to generate traditional canonical vortices, to optimize the separation of the resulting Fresnel diffraction orders. The other beam was used as a reference wave. The beam after the holographic mask was spatially filtered to extract the diffraction order containing the single-charge vortex and then passed through a lens of $10 \mathrm{~cm}$ focal length. The wave front of the resulting beam was analyzed by superposing the beam with the vortex nested and the reference wave tilted slightly relative to the propagation axis. The interference pattern arising exhibits a characteristic fork at the position of the dislocation and its topological charge is visually evaluated. The pattern of output light was analyzed by a chargecoupled device (CCD) camera. Noncanonical vortex dynamics were generated by operating the system with a 
cylindrical lens. The left and central rows of Fig. 3 show the observed outcome at different planes after the lens. As the ellipticity of the beam increases while it focuses, the vortex core becomes increasingly eccentric. At a crucial instant of the evolution the vortex converts itself into what, according to Figs. $2 \mathrm{c}$ and $2 \mathrm{~d}$, is believed to be a transverse vortex trajectory which inside the finite experimental accuracy always appears as an edge-line dislocation. Subsequently the quasiedge is observed to transform itself into a vortex of opposite topological charge relative to the input value. The orientation of the edge line in the plane is dictated by the value of the noncanonical strength. Notice that the inversion of the helicity of the spiral phase front of the vortex field is fully consistent with the imaging properties of the lens. We verified that the charge of a traditional canonical vortex does not vary by operating the system with a spherical lens (Fig. 3, right row).

We note the one-to-one map existing between the astigmatic scheme employed in the experiment and the evolution displayed in Fig. 2a. We stress that the observed inversion of the topological charge of the vortex does not occur at the surface of the lens, i.e., it does not involve a wave front dislocation induced by external discontinuities. We also stress that it occurs in free space, thus it is not related to any instabilities mediated with nonlinear effects or vortex solitons [10]. Rather, on the light of the relationship between general screw and edge dislocations studied by Nye [9], and of the generic vortex trajectories reported by Freund [8], it is believed to be a genuine and fundamental dynamic self-effect of noncanonical vortices.

Because of the associated spiral wave front, paraxial light beams with nested vortices carry orbital angular mo- mentum [12] that can be transferred to trapped suitable material particles causing them to rotate [5]. The total angular momentum can also contain a spin contribution, associated with polarization [13]. In the case of canonical vortices nested in symmetric beams, the orbital angular momentum, in units of $\hbar \omega$, is given by $L=m N$, where $m$ is the topological charge of the vortex and $N$ is the number of photons. In our experiment $m=1$, thus for the beam incident upon the lens $L / N=1$. We verified that in spite of the inversion of the topological charge of the vortex, the angular momentum of the beam is conserved during its evolution. Such angular momentum could be modified by passing the noncanonical vortex through additional optical elements [14], but otherwise in free space $L$ is constant so that $L / N=1$ everywhere. Therefore, contrary to traditional views, beyond the point where the charge of the vortex is inverted, the orbital angular momentum carried by the beam and the topological charge of the vortex nested in it have opposite signs. This fact has a simple interpretation. The beam incident upon the cylindrical lens is an almost pure Laguerre-Gaussian mode. The astigmatic lens redistributes the incoming orbital angular momentum into a superposition of Laguerre-Gaussian modes carrying different charges (Fig. 4), while keeping the total value constant. Therefore, the density of angular momentum is dynamically redistributed across the beam during its evolution.

We now consider the implications of the above to quantum photon states. The Laguerre-Gaussian modes form a complete Hilbert set and can thus be used to represent the quantum photon states within the paraxial theory of light propagation. The quantum angular momentum
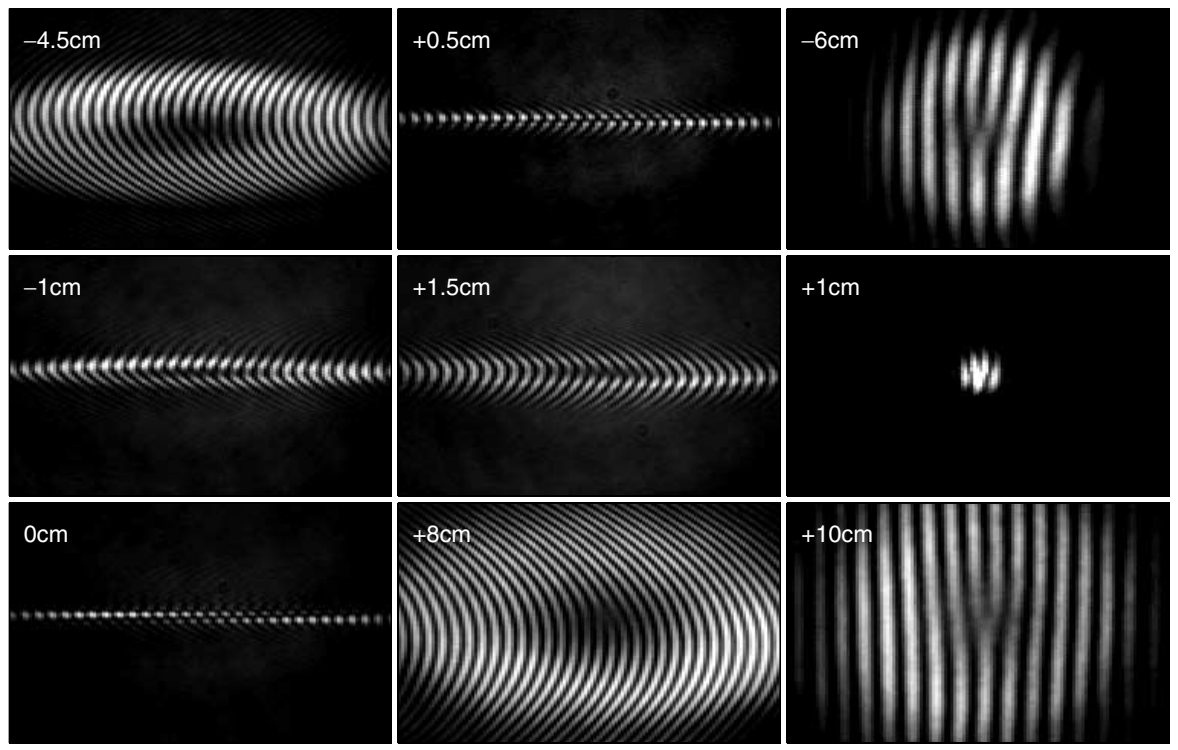

FIG. 3. Observed evolution of the topological charge of a vortex during a noncanonical evolution. The pictures show the evolution on propagation of the interference between the vortex beam and a reference beam tilted relative to the propagation axis. The images were acquired by a CCD camera placed at different positions beyond the lens. Labels inside the pictures stand for the location of the CCD camera relative to the lens focal plane. Left and center rows: Experimental realization of the noncanonical evolution generated by an input canonical vortex nested in a radially symmetric Gaussian beam with a $5 \mathrm{~mm}$ waist, passed through a cylindrical lens with a $10 \mathrm{~cm}$ focal length. Right row: Observed light pattern under identical conditions but with a spherical lens. 

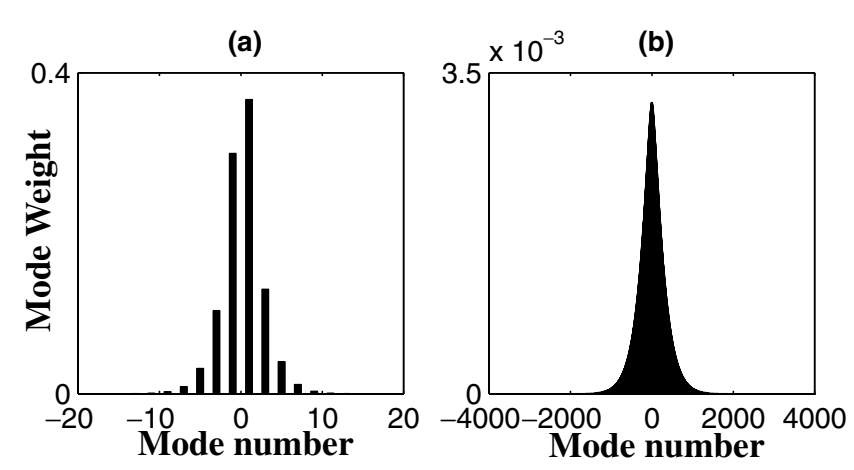

FIG. 4. Decomposition into normal modes with a well-defined angular momentum. Modes are numbered according to their topological charge, or azimuthal index. (a) Mode decomposition for the beam parameters of Fig. 2a. (b) Mode decomposition for the light beam with the noncanonical vortex nested used in the experimental observations, revealing that it contains $10^{3}$ modes.

number carried by the photon is then represented by the charge, or azimuthal index $m$, of the corresponding mode. The photons incident upon the lens are in an almost pure angular momentum state $|m=1\rangle$, but after the lens the photon state is a superposition of orbital angular momenta $|m\rangle$ (Fig. 4). The outcome is that, even though a full quantum average over many realizations yields the classical expectation $\langle L\rangle / n=1$, with $n$ the mean photon number, the quantum angular momentum number obtained in most of the realizations is not $m=1$. We thus notice the direct implications to the generation and teleportation of quantum entangled photons with angular momentum $[15,16]$ by parametric down-conversion of light in quadratic nonlinear crystals pumped with noncanonical vortex beams [23].

We finally point out that our observations here showing vorticity transformations of complex fields through noncanonical vortex trajectories and edge dislocations may provide new insight on the issue of how vorticity enters a different physical system governed by similar evolution equations. In particular, they may illuminate an old issue in superfluidity $[17,18]$, namely, how do quantized vortices enter into weakly interacting Bose-Einstein condensates trapped in a container that is stirred or rotated $[19,20]$. Our observations on the self-transformation of noncanonical vortices open up a new broad scenario where general dislocations, including edge dislocations, might evolve dynamically from the edge of the container towards the interior where the vortices are stabilized by repulsive many-body nonlinearities. They also suggest the possibility of dynamic charge inversion of a weakly interacting Bose-Einstein condensate vortex under the action of an asymmetric trapping potential.

In conclusion, we emphasize that the observed selfinversion of the topological charge of the vortex occurs through its dynamic propagation, and it does not involve discontinuities or nonlinearities or the nucleation of twin vortex pairs. Our observations reported here were performed with vortices nested in light beams, but the phe- nomenon is believed to be a generic effect of noncanonical vortices, thus the results have implications to all systems employing noncanonical vortex entities.

This work was supported by the Generalitat de Catalunya and by TIC2000-1010.

[1] R. Thom, Structural Stability and Morphogenesis (Benjamin, Reading, MA, 1975).

[2] J. F. Nye and M. V. Berry, Proc. R. Soc. London A 336, 165 (1974).

[3] Physics of Defects, edited by R. Balian, M. Kleman, and J.-P. Poirier (North-Holland, Amsterdam, 1981).

[4] A. T. Winfree, The Geometry of Biological Time (Springer, Berlin, 1990).

[5] A. Ashkin, Opt. Photonics News 10, 41 (1999); M.E. J. Friese et al., Nature (London) 394, 348 (1998); M. J. Padgett and L. Allen, Contemp. Phys. 41, 275 (2000).

[6] I. Freund, Opt. Commun. 159, 99 (1999).

[7] M. V. Berry, in Singular Optics, edited by M.S. Soskin, SPIE Proceedings Vol. 3487 (SPIE-International Society for Optical Engineering, Bellingham, WA, 1998), pp. 1-5.

[8] I. Freund, Opt. Commun. 181, 19 (2000); Opt. Lett. 26, 545 (2001); I. Freund and D. A. Kessler, Opt. Commun. 187, 71 (2001).

[9] J. F. Nye, Proc. R. Soc. London 378, 219 (1981).

[10] G. A. Swartzlander and C. T. Law, Phys. Rev. Lett. 69, 2503 (1992); A. W. Snyder, L. Poladian, and D. J. Mitchell, Opt. Lett. 17, 789 (1992); G. S. McDonald, K. S. Syed, and W. J. Firth, Opt. Commun. 94, 469 (1992).

[11] V. Yu. Bazhenov, M. V. Vasnetsov, and M. S. Soskin, JETP Lett. 52, 429 (1991); N. R. Heckenberg et al., Opt. Lett. 17, 221 (1992).

[12] L. Allen, M. J. Padget, and B. Babiker, in Progress in Optics, edited by E. Wolf (Elsevier, Amsterdam, 1999), Vol. XXXIX, pp. 291-372.

[13] R. A. Beth, Phys. Rev. 50, 115 (1936).

[14] E. Abramochkin and V. Volostnikov, Opt. Commun. 83, 123 (1991); S. J. van Enk and G. Nienhuis, Opt. Commun. 94, 147 (1992); M. W. Beijersbergen et al., Opt. Commun. 96, 123 (1993).

[15] A. Mair and A. Zeilinger, "Entangled States of Orbital Angular Momentum," http://info.uibk.ac.at/c/c7/c704/qo/ photon/_vortices

[16] A. Furusawa et al., Science 282, 706 (1998).

[17] S. J. Putterman, M. Kac, and G. E. Uhlenbeck, Phys. Rev. Lett. 29, 546 (1972).

[18] R. J. Donnelly, Quantized Vortices in Hellium II (Cambridge University Press, Cambridge, 1991).

[19] D. A. Butts and D. S. Rokhsar, Nature (London) 397, 327 (1999); J. E. Williams and M. J. Holland, Nature (London) 401, 568 (1999).

[20] M. R. Matthews et al., Phys. Rev. Lett. 83, 2498 (1999); K. W. Madison et al., Phys. Rev. Lett. 84, 806 (2000).

[21] G. Molina-Terriza, E. M. Wright, and L. Torner, Opt. Lett. 26, 163 (2001).

[22] Y. S. Kivshar et al., Opt. Commun. 152, 198 (1998).

[23] P. Di Trapani et al., Phys. Rev. Lett. 81, 5133 (1998); J. Arlt et al., Phys. Rev. A 59, 3950 (1999). 